\title{
Interaction of Insulin and Polymer Surface Investigated by Surface-MALDI-TOF-Mass Spectrometry
}

Zahida Ademović, ${ }^{1, *}$ Jochen Salber, ${ }^{2}$ and Doris $\mathrm{Klee}^{3}$

\footnotetext{
1 Faculty of Technology, University of Tuzla, Univerzitetska 8, 75000 Tuzla, Bosnia and Herzegovina

2 Chirurgische Klinik und Poliklinik, BG Universitaetsklinikum Bergmannsheil, Ruhr-University Bochum, Germany

3 Department of Textile Chemistry and Macromolecular Chemistry, RWTH Aachen, Pauwelsstr. 8, 52074 Aachen, Germany

* Corresponding author's e-mail address: zahida.ademovic@untz.ba
}

RECEIVED: July 14, 2014 * REVISED: February 23, 2015 * ACCEPTED: April 17, 2015

\begin{abstract}
Synthetic materials in contact with protein containing solution adsorb a considerable amount of proteins. The adsorption behaviour of zinc-free porcine insulin on the hydrophobic poly(vinylidene fluoride) (PVDF) surfaces before and after chemical vapour deposition (CVD) modification was directly analysed by matrix-assisted laser desorption ionisation-time-of-flight-mass spectroscopy in surface mode (surfaceMALDI-TOF-MS). The MALDI mass spectra of Zn-free porcine insulin dissolved in carbonate buffer pH 8.3 after adsorption onto non-modified and modified PVDF-CVD surfaces contain peaks assigned to monomer ion peak as well as peaks that are results of degradation of $\mathrm{Zn}$-free porcine insulin. The degradation is caused by structural changes taking place during adsorption of insulin onto hydrophobic surfaces and by subsequent laser induced desorption and ionisation process. Surface spectra of Zn-free porcine insulin dissolved in deionised water show only monomer ion peaks of porcine insulin without degradation product detected. Structure stability of $\mathrm{Zn}$-free porcine insulin upon adsorption is influenced by hydrophobic interaction between insulin and the surface.
\end{abstract}

Keywords: insulin, protein adsorption, biomaterials surface, MALDI-TOF-MS.

\section{INTRODUCTION}

M ANY synthetic surfaces adsorb proteins when exposed to protein-containing solutions. These interface-related phenomena is important to understand the processes taking place in, for example, chromatographic separation of proteins, detection assays for biomolecules, fouling of membranes, compatibility of biomaterials and protein aggregation. ${ }^{[1,2]}$ Also, materials used for administration of proteins in pharmaceutical dosage forms adsorb a considerable amount of protein and thus poses a risk factor for unsteady dosage of proteins. ${ }^{[3]}$ The protein adsorption process is complex and is still not fully understood. The rate and amount of adsorbed protein are dependent on the physico-chemical properties of the polymeric surface such as wettability and surface charge density. In general, adsorption behaviour of proteins is governed by electrostatic and hydrophobic interactions between protein and the solid surface. ${ }^{[4,5]}$ Proteins adsorbed on surfaces may undergo structural changes upon adsorption and the adsorption process is crucial for biocompatibility of the synthetic material. Therefore surface modification procedures are developed to create ultrathin interfaces at the implant surfaces which will control protein adsorption process.

Poly(vinylidene fluoride) (PVDF) has been employed as a biomaterial due to its nontoxicity, extraordinary durability and chemical stability. ${ }^{[6]}$ The coating of PVDF by chemical vapour deposition (CVD) polymerisation process using functionalised paracyclophane enables the introduction of functional groups (e.g. amino groups) in a single solventfree step. The understanding of the interaction which takes place between the modified PVDF surface and the components of the biological system is an important requirement of biomaterial development.

A number of techniques are available for studying protein adsorption onto polymer surfaces like optical methods $[7,8]$ 
and radiolabelling methods. ${ }^{[9,10]}$ For these techniques a large amount of proteins and a highly specific surface area are necessary to measure adsorbed proteins and receive enough signals. A method allowing the direct analysis of proteins adsorbed on surfaces would therefore be more useful. Molecular ions of complex molecules can readily be observed in the mass spectra obtained by the technique of matrix-assisted laser desorption ionisation (MALDI) in the time-of-flight (TOF) mode. MALDI can determine the molecular weight of proteins up to a few hundred kilodaltons ${ }^{[11]}$ with a high mass resolution and very low detection limits. ${ }^{[12]}$ Standard MALDI-TOF-MS spectra have been obtained after an analyte is dispersed at low concentration in a photoabsorbing matrix and are co-crystallized with it. ${ }^{[13]} \mathrm{A}$ laser shot decomposes the layer of matrix and eject the proteins which are ionized. The ionization, usually obtained by protonation, allows to accelerate the protein ions and to measure their mass to charge ratio. MALDI-TOF-MS in surface mode has been developed to detect molecular ions of analytes which are adsorbed onto material surface. ${ }^{[14]}$ MALDITOF-MS offers one of the simplest, rapid and most efficient methods for the analysis of surface-bound proteins.

The main interest in studying adsorption of insulin onto interfaces has been based on the use of the protein in various drug delivery systems to obtain controlled release administration, where the major focus has been on interactions of insulin with solid/liquid interfaces occurring in pump devices and on prevention of fibrillation. ${ }^{[15]}$ The protein conformation can be altered upon adsorption which in turn affects the biological function of the protein. Among other physical interactions responsible for protein adsorption, it is often found that hydrophobic interactions have a distinct effect on protein conformation. ${ }^{[16]}$

In this study, the interaction of $\mathrm{Zn}$-free porcine insulin physically adsorbed onto solid PVDF polymer surfaces was studied. The adsorption behaviour of $\mathrm{Zn}$-free porcine insulin in aqueous and buffered solution on the polymer surfaces with different hydrophobicity was analysed.

\section{MATERIALS AND METHODS}

\section{Surface Preparation}

Poly(vinylidene) fluoride (PVDF) was purchased from Solvay Adv. Polym. (France). PVDF granulate was pressed at $190^{\circ} \mathrm{C}$ for $10 \mathrm{~min}$ to obtain films and purified by Soxhlet extraction with hexane/ethanol.

The coating of PVDF by chemical vapour deposition (CVD) polymerisation process using functionalised paracyclophane was carried out in a self-designed machine by the method described elsewhere. ${ }^{[17]}$ Briefly the monomer 4Amino[2.2]-paracyclophane was pyrolyzed at $650{ }^{\circ} \mathrm{C}$ under reduced pressure and than condensed at $18^{\circ} \mathrm{C}$ on PVDF. After the procedure we had surfaces coated with poly[oamino- $p$-xylylene-co-p-xylylene] (PVDF-CVD).

Contact angles were measured in ultrapure water using the sessile drop method at room temperature on a G40 system (Krüss, Germany).

\section{Insulin Adsorption}

Porcine insulin was obtained from Aventis (USA) and was cleared from zinc using gel chromatography (Sephadex G 25). Zinc free insulin was dissolved in a $0.1 \mathrm{M}$ carbonate buffer $\mathrm{pH} 8.3$ or in deionised water to an end concentration of $1 \mathrm{mg} / \mathrm{ml}$. Surfaces were incubated in insulin solution for $1 \mathrm{~h}$ at $37{ }^{\circ} \mathrm{C}$ and rinsed twice with carbonate buffer and twice with deionised water. Surfaces incubated in deionised water were rinsed only with deionised water to remove weakly and non-adsorbed insulin. Reference surface spectra were obtained by incubating nonmodified PVDF in insulin solution for $1 \mathrm{~h}$ at $37{ }^{\circ} \mathrm{C}$ without subsequent washing. Finally, surfaces were dried under ambient conditions.

\section{Surface-MALDI-TOF-MS Analysis}

Surface-MALDI-TOF-mass spectra were obtained using a

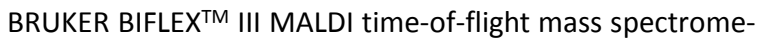
ter (Bruker-Franzen Analytik, Germany) equipped with a SCOUT-ion source, an ion mirror (reflectron) and standardmicrochannel-plate-detectors. The instrument was used with a variable accelerating voltage up to $20 \mathrm{kV}$ and operating in reflection mode. A pulsed nitrogen laser (337 nm wavelength and 3 ns pulse width) was used for matrix crystal deposition. The laser power was adjusted between $50-70 \%$ of its maximum. For each spectrum $100-150$ shots were summed. The instrument control and data processing were accomplished with the software Bruker XTOF 3.1.0 supplied by Bruker.

In present experiments a small sample piece cut from PVDF and PVDF-CVD surfaces after adsorption with insulin were placed onto the stainless steel MALDI sample holder. Sinapinic acid in a $0.1 \%$ solution of trifluoroacetic acid in acetonitrile/water was applied onto the sample surface. Before inserting the sample holder into the spectrometer the solvent was evaporated. Thereafter, pulsed UV laser irradiation causes volatilisation of matrix crystals and the embedded protein molecules, which were ionised, extracted and detected in standard MALDI-MS fashion.

\section{RESULTS AND DISCUSSION}

Porcine insulin is a small globular protein with a molecular mass of $5777 \mathrm{Da}$ and is composed of a A-polypeptide chain (21 amino acids) which contains an intrachain disulfide bond and is linked to the B-polypeptide chain (30 amino acids) 


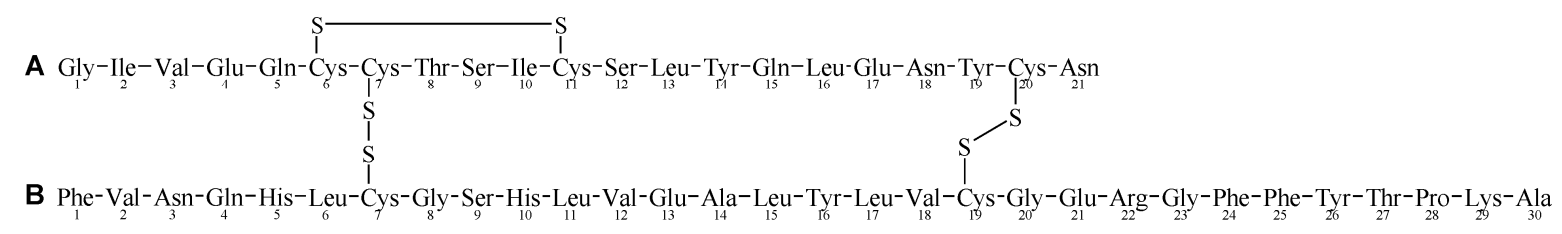

Figure 1. The chemical sequence of the porcine insulin.

which contains two interchain disulfide bonds. The full amino acid sequence of porcine insulin is shown in Figure 1.

Crystallographic studies indicate that insulin contains three $\alpha$-helices (two located in the A-chain at position A1-A9 and A12-A19 and one located in B-chain at position B9-B19).[18] However, the insulin structure is highly dynamic, with the $\mathrm{N}$-terminal (B1-B8) and $\mathrm{C}$-terminal segments (B25-B30) of the B-chain having the greatest flexibility ${ }^{[19]}$ (Figure 2).

The insulin monomer has nonpolar amino acids exposed to the medium, which is not thermodynamically favorable and the equilibrium constant for the formation of dimers is high. ${ }^{[20]}$ The dimer is generally recognized as the predominant starting species. At higher insulin concentrations three dimers joins together into a hexamer. Other factors that affect the self-association of insulin are $\mathrm{pH}$, ionic strength and the presence of certain divalent ions. When the two monomers are associated a shared antiparallel $B$ sheet structure is formed between the two $\mathrm{C}$-terminal strands of the B-chain. ${ }^{[22]}$ This shared structure makes the monomer-monomer interaction stronger than the interactions involved in the formation of tetrameric and hexameric

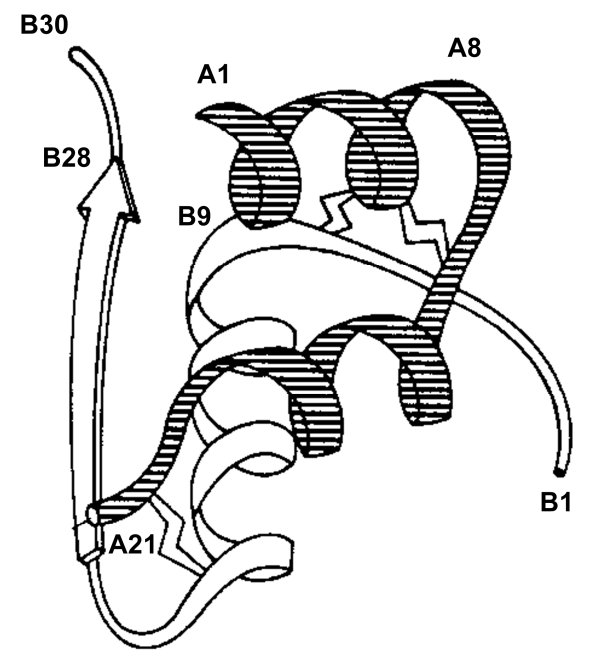

Figure 2. Ribbon drawing of the monomeric porcine insulin secondary structure, including disulfide bonds. A-chain is striped. Helices are spirals, the beta sheet is an arrow and loops are lines. states of insulin. The formation of the hexamer results in the burial of nonpolar amino acids within the core of the hexamer. Those nonpolar amino acids are otherwise exposed on the surface of the dimer. The hexamer is therefore the most hydrophilic species of insulin.

The multimerization and aggregation of insulin are of great importance in biology, pharmacology, and protein fibrilogenesis. Insulin is stored in the pancreas as the inactive zinc hexamer. When released into the blood serum by the $\mathrm{pH}$ change, this hexamer dissociates into a dimer and then subsequently into a monomer, which is its physiologically active form. However, the insulin monomer is less stable than the hexamer and tends to aggregate. Under physiological conditions, $\mathrm{Zn}$-insulin shows reduced fibril formation relative to $\mathrm{Zn}$-free insulin because of the formation of the stable $\mathrm{Zn}$-insulin hexamer. ${ }^{[23]}$ The effect of $\mathrm{Zn}^{2+}$ on multimer equilibria is dramatic because of the formation a stable $\mathrm{Zn}$ insulin complex, thought to arise from the combination of two $\mathrm{Zn}$ ions with three native conformation insulin dimers. ${ }^{[24]}$

Insulin interacts weakly with hydrophilic surfaces, whereas stronger interaction are present with hydrophobic surfaces at neutral $\mathrm{pH} .{ }^{[25]}$ The first stages in the adsorption process involve transport of the protein to the surface, deposition and subsequently unfolding or spreading of the protein to thermodynamically more stable conformations. Since the monomeric and dimeric forms are the most hydrophobic species, they are expected to be the species most prone to adsorption. The monomer is the least stable species in solution and that interfacial aggregation is initialized by adsorption of the monomer. ${ }^{[26]}$ Other insulin species interact with the interface in a reversible manner, but the monomer may unfold irreversibly at the interface, which could be the first step in the aggregation pathway.

In present work, surface-MALDI-TOF-MS was used to directly analyse porcine insulin that was physically adsorbed onto PVDF surfaces before and after CVD modification. These surfaces differ in hydrophobicity. The contact angle measurement by sessile drop method shows the hydrophobic nature of both PVDF surface. Contact angle of untreated PVDF is $83^{\circ}$ and this value increase to $92^{\circ}$ after modification of the surface using CVD polymerisation process. This increasing is caused by aromatic rings from deposited CVD polymer layer. 
Figure 3 shows the positive MALDI spectra of 1 $\mathrm{mg} / \mathrm{ml} \mathrm{Zn}$-free porcine insulin dissolved in carbonate buffer pH 8.4 and subsequently adsorbed onto different PVDF surfaces. All spectra showed a strong protonated monomer ion peak (1) at $m / z$ value 5745 which corresponds to protonated monomer ion $[\mathrm{M}+\mathrm{H}]^{+}$. This peak is joined on the right side by a small adduct peak (2) created by the binding of insulin with a matrix molecule. Also, an insulin double charged monomer ion peak (4) at $\mathrm{m} / \mathrm{z}$ value 2880 is observed, which corresponds to $[\mathrm{M}+2 \mathrm{H}]^{2+}$. Interestingly, in Figures $3 b$ and $3 c$ an additional peak (3) is shown which is not detected in the reference surface spectrum of porcine insulin (Figure 3a). Additionally, in the spectrum of porcine insulin adsorbed on PVDF-CVD surface other peaks (5), (6) and (7) are detected (Figure $3 \mathrm{c}$ ).

The determination of the mass difference between the monomer ion peak (1) and degradation peak (3) varies generally between 260 and $310 \mathrm{Da}$. The mass difference between double charged ion peak (4) and degradation peak (5) ranges from 130 to $260 \mathrm{Da}$. The peaks (6) are detected between 4200 and $4300 \mathrm{Da}$ and peak (7) between 4750 and $4830 \mathrm{Da}$. The exact determination of $\mathrm{m} / \mathrm{z}$ of the additional degradation peaks is not possible as the variations in sample preparation and the instrumental resolution resulted in a broadening of mass peaks of insulin samples. ${ }^{[27]}$ Additionally, MALDI measurements in surface mode of biomolecules is performed on solid carriers of undefined thickness, therefore the mass scale is shifted because the TOF detector is calibrated for sample analysis using standard sample holders. By sticking a piece of PVDF onto the sample holder, the ion path is slightly shorter, which causes an indeterminable shift in the $m / z$ classification. ${ }^{[14]}$

It is likely that sensitive MALDI detection of adsorbed biomolecules depends on desorption of analyte from the surface by matrix and at the same time an effective incorporation of the analyte molecules into the matrix crystals. In the standard MALDI technique protein analytes are dispersed in solution together with matrix molecules and consequently good mixing and incorporation of the analyte into the growing matrix crystals after evaporation of the solvents is achieved. In the MALDI-surface mode, matrix molecules are added on the protein (analyte) layer that is adsorbed on the polymer surface. Therefore, protein molecules need to be desorbed from the surface and incorporated into the growing matrix crystals. Because protein molecules must be first desorbed off the solid surface and then dispersed in the matrix crystals, the interaction between protein and solid surface as well as interaction between protein and matrix must be considered. If the matrix is not able to desorb and encapsulate protein molecule completely, some part of protein molecule remains unprotected by matrix and is exposed to laser energy. It should be taken in account that only the most tightly bound insulin
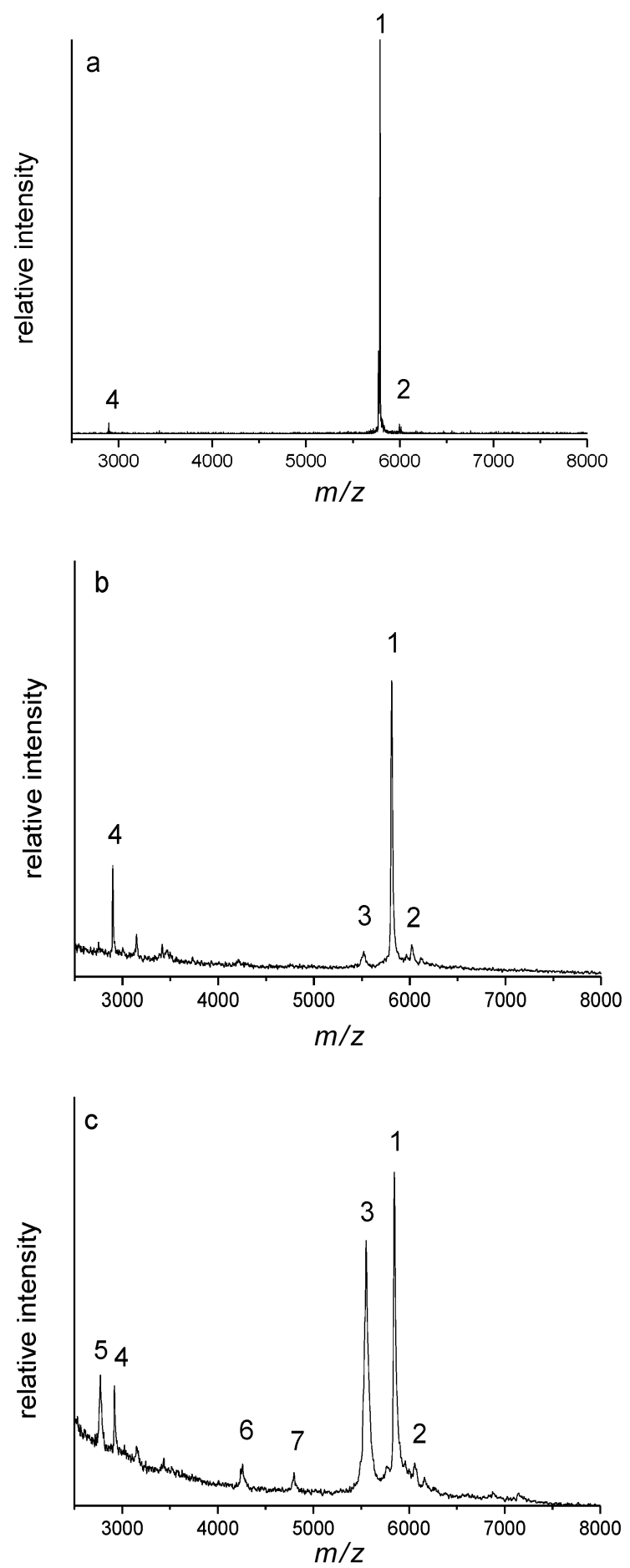

Figure 3. MALDI-TOF mass spectra of $1 \mathrm{mg} / \mathrm{ml} \mathrm{Zn-free}$ porcine insulin in carbonate buffer $\mathrm{pH} 8.3$ adsorbed on a) PVDF without washing (reference), b) insulin adsorbed on PVDF surface and c) insulin adsorbed on PVDF-CVD surface. Spectra: peak (1) - protonated monomer ion $[\mathrm{M}+\mathrm{H}]^{+}$, peak (2) - binding of insulin with a matrix molecule, peak $(3,5,6$, 7) - degradation peaks, peak (4) - insulin double charged monomer ion $[\mathrm{M}+2 \mathrm{H}]^{2+}$. 

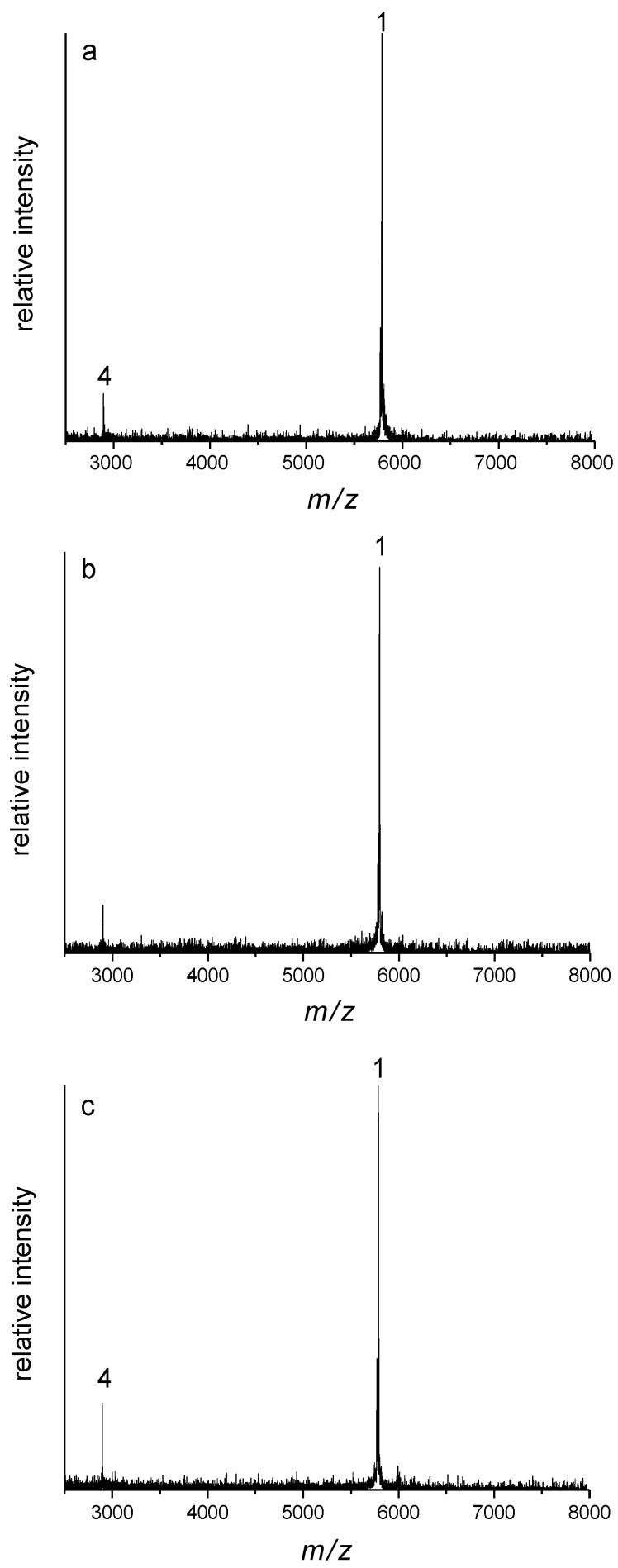

Figure 4. MALDI-TOF mass spectra of $1 \mathrm{mg} / \mathrm{ml} \mathrm{Zn}$-free porcine insulin in deionised water $\mathrm{pH} 4.3$ adsorbed on a) PVDF without washing (reference), b) insulin adsorbed on PVDF surface and c) insulin adsorbed on PVDF-CVD surface. Spectra: peak (1) - protonated monomer ion $[\mathrm{M}+\mathrm{H}]^{+}$, peak (4) - insulin double charged monomer ion $[\mathrm{M}+2 \mathrm{H}]^{2+}$. molecules in Figure $3 \mathrm{~b}$ and $3 \mathrm{c}$ were analysed because less strongly adsorbed insulin were desorbed and washed away before analysing the samples. Some studies have demonstrated that strongly surface-bound peptides are inefficiently ionised in MALDI experiments, presumably due to the incomplete and poor incorporation of the protein into the MALDI matrix crystals. ${ }^{[28]}$

The spectra of porcine insulin adsorbed on PVDF surface (Figure 3b) shows only a weak degradation peak (3) in comparison to PVDF-CVD surface (Figure 3c). On PVDF-CVD surface in addition to a strong degradation peak (3), the peaks (4), (5) and (6) are observed. These peaks belong to degradation products of porcine insulin caused by MALDI laser-induced desorption and ionisation process. Porcine insulin molecules are strongly adsorbed on the hydrophobic surface and are incapable to desorbs and incorporate in the crystal matrix during MALDI desorption and ionisation process. This implies that the insulin structure is largely destabilised upon adsorption onto hydrophobic surfaces, thus illustrating the general observation that hydrophobic interactions have a relatively strong effect on the protein conformation. The strong interaction of insulin with the hydrophobic surface is accompanied by a strong reduction in its structural stability compared to the stability in solution. ${ }^{[24]}$ This effect is more pronounced for more hydrophobic PVDF-CVD surface in comparison to less hydrophobic PVDF surface.

Regarding the mass differences between the peaks, the degradation products that belong to the peak (3) and peak (5) could come from either the first three amino acids at the $\mathrm{N}$-terminus of the $\mathrm{A}$-chain or the last three amino acids at the C-terminus of the B-chain, assuming that these sequences were cleaved during laser desorption and ionisation process. The three hydrophobic amino acids A1-A3 (Gly-lle-Val) are in a relative fixed conformation state regarding to their affiliation to the helical part of the $\mathrm{N}$-terminus of the A-chain. It is very probably that the strong hydrophobic amino acids B24-B26 (Phe-Phe-Tyr) in the beta sheet forming sequence (Figure 1) adsorb onto the hydrophobic solid surface to form very strong hydrophobic interaction. The high flexible and hydrophilic $\mathrm{C}$-terminus of the B-chain (B28-B30) remains free (Figure 2). Consequently the MALDI matrix solution is not able to desorb completely the porcine insulin molecule and thus co-crystallisation of the whole porcine insulin molecule with matrix molecules is not ensured. Presumably a part of insulin is incomplete incorporated in the matrix. We postulated here that the flexible part of B-chain (B28-B30) remains unprotected by matrix and is exposed to laser energy. The high intensity of laser energy breaks the peptide bond between B27 and B28. The resulting degradation product is seen in Figure $3 \mathrm{~b}$ and $3 \mathrm{c}$ as peaks (3). The peaks (6) and (7) are not 
characterized yet. Further investigation is required to affirm our assumption and to identify the non characterised peaks. In the non-washed reference surface spectrum (Figure $3 a$ ) the peaks of the degradation products are not detected, because porcine insulin adsorbed from a $1 \mathrm{mg} / \mathrm{ml}$ solution forms a multilayer onto the PVDF surface. Therefore, only less bound insulin molecules incorporated in multilayer coverage of the surface that are not directly adsorbed to the hydrophobic surface are desorbed and were able to completely associate with matrix crystals during preparation.

Examples of the positive MALDI mass spectra of 1 $\mathrm{mg} / \mathrm{ml} \mathrm{Zn-free} \mathrm{porcine} \mathrm{insulin,} \mathrm{which} \mathrm{was} \mathrm{dissolved} \mathrm{in} \mathrm{de-}$ ionised water and subsequently adsorbed onto the PVDF and PVDF-CVD surfaces, are shown in Figure 4 . In contrast to Figure 3 all spectra display a strong monomer ion peak (1) corresponding to $[\mathrm{M}+\mathrm{H}]^{+}$and a porcine insulin double charged ion peak (4) which corresponds to $[\mathrm{M}+2 \mathrm{H}]^{2+}$. No degradations peaks are detected.

The insulin behaviour in solution at different concentration and $\mathrm{pH}$ could explain these results. Insulin exists in equilibrium between monomer, dimer and hexamer. The degree of association is highly influenced by the concentration and type of insulin. This may also influence stability and especially adsorption. It has been shown that the $\mathrm{Zn}$-free human insulin at concentrations above $1 \mathrm{mg} / \mathrm{ml}$ is in a $70 \%$ dimer and $30 \%$ monomer form. At concentrations below 0.1 $\mathrm{mg} / \mathrm{ml}$, at $\mathrm{pH} 7.4$ only monomers are found. ${ }^{[29]}$ Other have observed mainly monomers below concentrations of 3 $\mathrm{mg} / \mathrm{ml}$ for various insulin variants. ${ }^{[30]}$ The structure would be expected to vary if the protein adsorbed to the surface was either monomer or a combination of monomer, dimer or even hexamer. The packing and the available space on the surface to accommodate structural changes would vary, as would the ratio of monomer to dimer. The exposure of hydrophobic area at the protein surface also varies depending on the presence and distribution of monomer to dimer to hexamer and this in turn could influence both the adsorption and the structural changes upon adsorption. The structure of adsorbed protein does differ from that in solution, and many proteins also tend to form more $\beta$-sheet structure upon adsorption or lose structural elements such as $\alpha$-helix. ${ }^{[31,32]}$

In solution at neutral $\mathrm{pH}$ and at low concentration insulin exists as a monomer. ${ }^{[33]}$ At higher concentrations at acidic or neutral $\mathrm{pH}$ in absence of zinc the insulin monomer self-associates to form dimmers in which only intermolecular forces between the molecules are presented. ${ }^{[34]}$ It should be taken in account that the $\mathrm{Zn}$-free insulin used in this experiment contains a considerable amount of acetic acid so that the zinc-free insulin disolved in deionised water has an acidic $\mathrm{pH}$ 4.3. In addition, the concentration of the solution is very high $(1 \mathrm{mg} / \mathrm{ml})$. Therefore, we assume that porcine insulin under these condition forms dimers. The MALDI matrix solution is able to desorbs and completely encapsulate the porcine insulin molecules from dimer layer.

Monomer as well as dimer and hexamer can adsorb to the surface. It has been described that the insulin monomer is the least stable of these three conformations and is most likely to change conformation upon adsorption to a hydrophobic surface. ${ }^{[35]}$ Therefore, the monomer would undergo structural changes, whereas dimer and hexamer would not. Furthermore, it has been indicated that the monomer is the species with the highest tendency to adsorb to hydrophobic surfaces. ${ }^{[36]}$ Therefore, the distribution between monomer, dimer and hexamer, which is highly dependent on concentration, zinc content and type of insulin, could have an effect on adsorption. ${ }^{[37]}$

It is assumed that $\mathrm{Zn}$-free porcine insulin is adsorbed as monomer at $\mathrm{pH} 8.3$ and changes conformtion when adsorbed on high hydrophobic surfaces PVF-CVD. When Znfree porcine insulin is adsorbed at $\mathrm{pH} 4.3$, it forms dimers and is more stable specie that does not change conformation during adsorption process.

\section{CONCLUSION}

In this work a surface-MALDI-TOF-MS method for studying adsorption of zinc free porcine insulin onto different PVDF surfaces was described. By means of this technique it is possible to determine $\mathrm{Zn}$-free porcine insulin behaviour during adsorption onto material surfaces and to analyse structural stability of these surface bound molecules. The strong interaction of $\mathrm{Zn}$-free porcine insulin which was dissolved in a carbonate buffer $\mathrm{pH} 8.3$ with the hydrophobic surface is accompanied by a reduction in ability of the MALDI-matrix solution to desorb and encapsulate insulin molecules. As the result the degradations products of insulin were detected in surface spectra. The Zn-free porcine insulin adsorbed more strongly onto high hydrophobic PVDF-CVD surface in comparison to less hydrophobic PVDF surface. By dissolving $\mathrm{Zn}$-free porcine insulin in deionised water at high concentration, the protein molecules form dimer which, during MALDI procedure, are desorbed from the surface and incorporated in the matrix. Thus only the monomer ion peaks of porcine insulin were detected. However more detailed determinations and characterisations of the degradation products as well as association of insulin onto surfaces is necessary for a complete understanding of the adsorption, desorption and ionisation processes. The exact interaction between insulin and matrix molecules still remains to be resolved.

Acknowledgment. This work was supported by the Aachen Centre of Competence: biomaterials (bWA), RWTH-Aachen, BMBF-Project No. 03N4903. The authors thank Anita Verbovsek for the MALDI-TOF-MS measurements. 


\section{REFERENCES}

[1] Surface and Interfacial Aspects of Biomedical Polymers, Vol. 2 (Ed.: J. D. Andrade), Plenum, New York, 1985.

[2] S. Sharma, R. W. Johnson, T. Desai, Langmuir 2004, 20, 348 .

[3] M. Hewson, V. Nawadra, J. Oliver, C. Odgers, J. Plummer, K. Simmer, J. Paediatr. Child H. 2000, 3, 216.

[4] A. S. Hoffman, J. Biomater. Sci. Polym Edn. 1999, 10, 1011.

[5] R. D. Tilton, C. R. Robertson, A. P. Gast, Langmuir 1991, 7, 2710.

[6] G. Laroche, Y. Marois, R. Guidon, M. W. King, L. Martin, T. How, Y. Douville, J. Biomed. Mat. Res. 1995, 29, 1525.

[7] M. Balcells, D. Klee, M. Fabry, H. Höcker, J. Colloid Interface Sci. 1999, 220, 198.

[8] J. J. Remsden, J. Stat. Phys. 1993, 73, 853.

[9] A. Kidane, J. M. Szabocsik, and K. Park, Biomaterials 1998, 19, 2051.

[10] M. Holmberg, K. B. Stibius, N. Sokol, L. B. Niels, P. Kingshott, H. L. Xiaolin, Anal. Biochem. 2007, 361, 120.

[11] F. Hillenkamp, M. Karas, R. C. Beavic, B. T. Chait, Anal. Chem. 1991, 63, 1193.

[12] M. Karas, F. Hillenkamp, Anal. Chem. 1988, 60, 2299.

[13] O. Vorn, P. Roepstorff, M. Mann, Anal. Chem. 1994, 66, 3281.

[14] P. Kingshott, H. A. W. St. John, H. J. Griesser, Anal. Biochem. 1999, 273, 156.

[15] Y. M. Kwon, M. Baudys, K. Knutson, S. W. Kim, Pharm. Res. 2001, 18, 1754.

[16] J. Buijs, V. J. Hlady, Colloid Interface Sci. 1997, 190, 171.

[17] J. Lahann, D. Klee, H. Hoecker, Macromol. Rapid Comm. 1998, 19, 441.
[18] T. Blundell, G. Dodson, D. Hodgkin, D. Mercola, Adv. Protein Chem. 1972, 26, 279.

[19] C. Chotia, A. M. Lesk, G. G. Dodson, D. C. Hodgin, Nature 1983, 302, 500.

[20] B. H. Frank, A. H. Pekar, A. J. Veros, Diabetes 1972, 21, 486.

[21] Y. Pocker, S. B. Biswas, Biochemistry 1981, 20, 4354.

[22] J. Brange, L. Langkjoer, Pharm. Biotechnol. 1993, 5, 315.

[23] N. Anda, G. Julia, S. Julia, T. Vello, P. Peep, Biochem. J. 2010, 430, 511.

[24] J. Brange, L. Adersen, E. D. Laursen, G. Meyn, E. J. Rasmussen, J. Pharm. Sci. 1997, 86, 517.

[25] P. Nilsson, T. Nylander, S. Havelund, J. Colloid Interface Sci. 1991, 144, 145.

[26] V. Sluzky, A. M. Klibanov, R. Langer, Biotechnol. Bioeng. 1992, 40, 895.

[27] J. Buijs, C. C. Vera, E. Ayala, E. Steensma, P. Hakansson, S. Oscarsson, Anal. Chem. 1999, 71, 3219.

[28] J. Zhang, G. R. Kinsel, Langmuir 2003, 19, 3531.

[29] L. Jorgensen, P. Bennedsen, S. V. Hoffmann, R. L. Krogh, C. Pinholt, M. Groenning, S. Hostrup, J. T. Bukrinsky, Eur. J. Pharm. Sci. 2011, 5, 509.

[30] L. Krogh, C. Pinholt, M. Groenning, S. Hostrup, and J. T. Bukrinsky, Eur. J. Pharm. Sci. 2011, 42, 509.

[31] C. E. Giacomelli, W. Norde, Biomacromolecules 2003, 4, 1719.

[32] A. Sethuraman, G. Belfort, Biophys. J. 2005, 88, 1322.

[33] A. H. Pekar, B. H. Frank, Biochemistry 1972, 11, 4013.

[34] P. D. Jeffrey, J. H. Coates, Biochemistry 1966, 5, 489.

[35] L. Nielsen, R. Khurana, A. Coats, S. Frokjaer, J. Brange, S. Vyas, V. N. Uversky, A. L. Fink, Biochemistry 2001, 40, 6036.

[36] T. Arnebrant, T. Nylander, J. Colloid Interface Sci. 1988, 122, 557.

[37] J. Donsmark, L. Jorgensen, S. H. Mollmann, S. Frokjaer, C. Rischel, Pharm. Res. 2006, 23, 148. 